\title{
Small-Scale Void-Size Determination in Reinforced Concrete Using GPR
}

\author{
Yong Yang, ${ }^{1,2}$ Junwei Lu, ${ }^{2}$ Rongzhe Li, ${ }^{3}$ Weigang Zhao $\left(\mathbb{D},{ }^{1,3}\right.$ and Deli Yan ${ }^{2}$ \\ ${ }^{1}$ State Key Laboratory of Mechanical Behavior and System Safety of Traffic Engineering Structures, \\ Shijiazhuang Tiedao University, Shijiazhuang 050043, China \\ ${ }^{2}$ School of Electrical and Electronic Engineering, Shijiazhuang Tiedao University, Shijiazhuang 050043, China \\ ${ }^{3}$ Structure Health Monitoring and Control Institute, Shijiazhuang Tiedao University, Shijiazhuang 050043, China \\ Correspondence should be addressed to Weigang Zhao; zhw0876@stdu.edu.cn
}

Received 17 December 2019; Accepted 20 February 2020; Published 6 June 2020

Academic Editor: Liborio Cavaleri

Copyright (c) 2020 Yong Yang et al. This is an open access article distributed under the Creative Commons Attribution License, which permits unrestricted use, distribution, and reproduction in any medium, provided the original work is properly cited.

\begin{abstract}
The detection and evaluation of void in concrete are imperative issues in health monitoring of civil engineering. However, the void is difficult to be detected at its early stage of formation on account of its small scale and concealment. Although, in view of the remarkable performance such as precision and continuity, ground penetrating radar (GPR) is widely employed in some special fields of nondestructive testing, the strong reflection of rebar which could submerge the echo of void is now a stumbling block for the application of GPR in reinforced concrete. In order to develop the tactic of small-scale void-size determination with considerable noise originated from rebar, a two-stage regression method is proposed in this study. First, a superposition model of target echoes was established to analyze the relationship between the space-time distribution and spectrum of the rebar echo and the void echo, and a curvelet transform was employed to reduce the strong reflection of the rebar. Then, the echo amplitude of small-scale void was investigated, and a regression method was put forward to determine the void size of small scale. Finally, forward simulation and experiment were carried out to verify the feasibility of the proposed method. Results demonstrated that the spectral distribution of the rebar echo is significantly higher than that of the void echo. The curvelet-based low-pass filter can effectively ensure the discrimination of void echo. The regression method performs a high-accuracy void-size determination with more than $90 \%$ in small scale.
\end{abstract}

\section{Introduction}

In view of the remarkable performance such as durability and bearing capacity, reinforced concrete (RC) is widely used in high-rise buildings, municipal projects, dams, tunnels, high-speed railways, and other structures. It is well known that, due to construction quality, external loads, and some other particular common factors, void would inevitably be formed and developed during the service period of $\mathrm{RC}$ structures. In the early stage of void formation, it is difficult to be detected because of its small scale and strong concealment. If left unchecked, the voids would destroy the integrity of the RC structure and accelerate the failure of the RC structure. Therefore, the fast and timely detection and accurate evaluation of internal defects in RC structures are imperative issues in health monitoring of civil engineering.

In the existing detection methods for RC structure internal defects, core drilling and nondestructive testing are most widely used. The core drilling method has the characteristics of high detection accuracy. However, the randomness of the sampling points makes it difficult to express the distribution of the defects in the whole region. Drill-core sampling is destructive to the structure, and it is not suitable for the fields, such as in high-speed railway ballastless tracks that require strict structural integrity. Nondestructive testing methods include impact echo $[1,2]$ and ground penetrating radar (GPR) [3, 4]. In the impact echo method, the detection precision is related to the ratio of the depth and void size, 
and the resolution is relatively poor. Additionally, the echo components of the elastic wave are complex, including $P$ waves and $S$ waves, which presents difficulties in signal processing. Slow detection speed is also an important factor that limits the application of this method.

GPR is an effective nondestructive testing method that is characterized by its rapidity, continuity, high detection accuracy, and ability to overcome the shortcomings of the core-sampling and impact echo methods. The unique advantage of GPR causes concern of many scholars.

Detection and size determination of underground targets are important aspects of GPR research. Ahmadi and Fathianpour studied the cross-correlation function of the target and the echo profile of GPR obtained from the target detection field. By establishing the echo template of the underground target, a method of pattern matching was used to detect the underground target [5]. Zanzi and Arosio obtained GPR echo data using two antennas with vertical and horizontal polarization, and they developed a method based on radar cross section (RCS) to determine the size of the steel bar [6]. Additionally, Hoegh et al. used an aircoupled antenna array to detect voids in asphalt concrete [7]. He et al. proposed an $S$ transform-based method to detect and locate the thickness of a void in a thin layer [8]. Brian took advantage of GPR to detect a cavity inside an ancient building [9]. David analyzed the "bright spot" characteristics of the cavity's echo response [10].

For target size determination, Mechbal and Khamlichi used a hyperbolic fitting method to obtain the fixed point of the wave velocity and the vertex of the hyperbola. They then proposed an optimal regression theory to obtain the size of reinforcement [11]. Windsor et al. generalized the Hough transform to identify the size of an underground cylindrical pipeline. They also explored the buried pipe (depth $1 \mathrm{~m}$, diameter $0.18 \mathrm{~m}$ ) that had an estimated size of $0.174 \mathrm{~m} \mathrm{[12].}$ On the basis of the Welch power spectral density estimate, Nuaimy proposed a backpropagation neural network to classify the targets and then used the Hough transform on the edges of these reflections to accurately identify the depth and position of the buried targets [13]. Pasolli et al. used support vector machines and Gauss processing methods to classify limestone, metal, and air. The horizontal and vertical positions were identified with an accuracy of $82 \%[14,15]$.

These researchers have mainly been focused on the detection of underground pipelines and rebar in RC structures. This has rarely involved the detection and identification of void defects inside RC structures, especially small-scale void defects.

Hence, in recent decades, with advances in geometric multiscale analysis (GMA) algorithms like ridgelet and curvelet have been introduced to analyze the echo profiles of GPR [16-18]. Regarded as a directional filter, Tzanis utilized the curvelet transform to detect a layered structure $[19,20]$. However, his main work concentrated on large-scale layered structures; small-scale void detection in RC structures was not investigated.

This study focuses on small-scale void defect detection and determination in RC structures. The study is organized as follows. In Section 2, spectrum of multitarget echo is analyzed, and a regression-based method is advocated to determine the size of small-scale void defects. In Section 3, forward simulation and experiment are carried out to verify the proposed method, and analysis and discussion are conducted. In Section 4, the study is summarized and related conclusions are presented.

\section{Methodology}

Unlike target detection in plain concrete or soil, void detection and size determination in reinforced concrete have the additional obstacle of a strong rebar reflection. To reduce the strong reflection interference, target echo analysis is required.

\subsection{Target Echo Analysis}

2.1.1. Multitarget Echo Superposition Model. When GPR is used to detect a void in an RC structure, the echo profile mainly includes ground direct wave, rebar echo, void echo, and other clutter signals, as shown in Figure 1.

In Figure 1, $T$ and $R$ represent the transmitter and the receiver, respectively; $S_{r}$ represents the rebar echo; $S_{v}$ represents the void echo; $S_{d}$ represents the direct wave; and $S_{n}$ represents the clutter signals. When the diameter, $d$, of the rebar is small, and the interval between the rebar is far larger than $d$, the echo amplitude of the void defect is not disturbed by the rebar echo. Therefore, the profile of GPR can be expressed as

$$
S=S_{r}+S_{v}+S_{d}+S_{n}
$$

The GPR echo profile (also known as a $B$-Scan) is composed of multiple single measurements ( $A$-Scans). Given that the $i^{\text {th }}$ echo signal $A$-Scan is $S_{i}$, the $S$ can be expressed as

$$
S=\left\{S_{1}, S_{2}, \cdots, S_{m}\right\}
$$

where $S_{i}=S_{r i}+S_{v i}+S_{d i}+S_{n i}$.

So, the echo profiles of GPR are composed of different target echoes using additive superposition.

2.1.2. Space-Time Analysis of Target Echo. In a GPR echo profile of an RC structure, there are many methods to filter the direct wave on the ground, and these methods have achieved good results. In this study, by ignoring the random noise, $S_{n}$, and the direct wave, $S_{d}$, the target echo, $S$, can be simplified to the rebar echo, $S_{r}$, and the void echo, $S_{v}$.

(1) Rebar Echo Model. As shown in Figure 2(a), the rebar target can be regarded as a point target, and the rebar target echo equation can be expressed as

$$
S_{r}(x)=\frac{2}{v} \sqrt{x^{2}+z_{0}^{2}},
$$

where the intersection point between the right upper direction of the steel bar and the survey line is the coordinate origin $O(0,0)$. The survey direction is the positive direction of the transverse axis- $x$, and the downward direction is the 


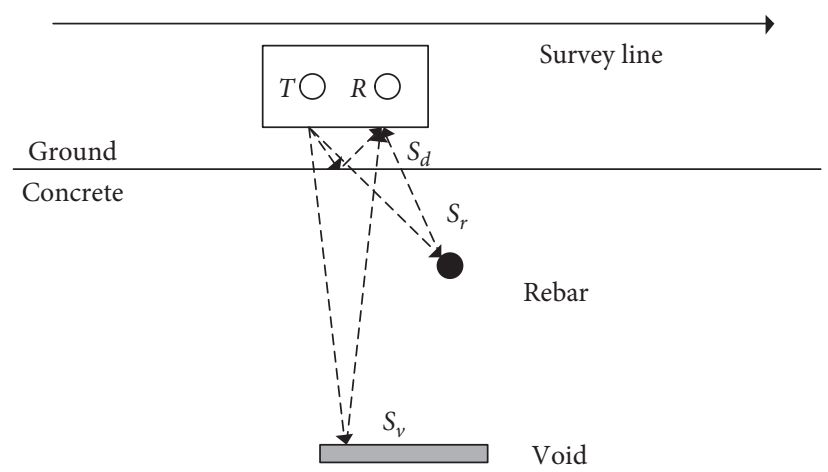

FiguRe 1: Multitarget echo submerged model.

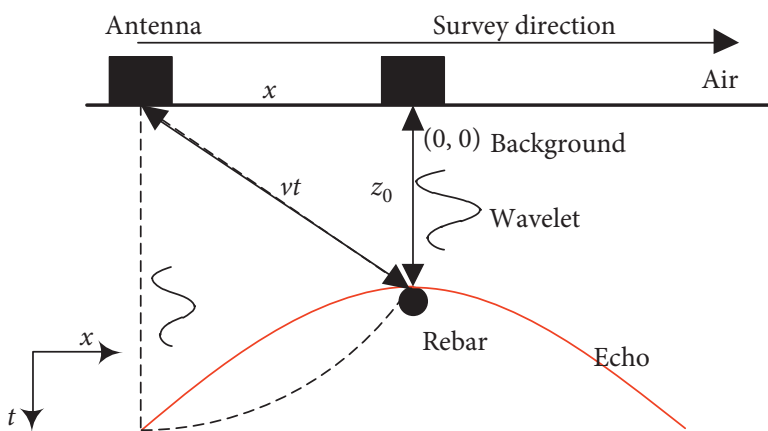

(a)

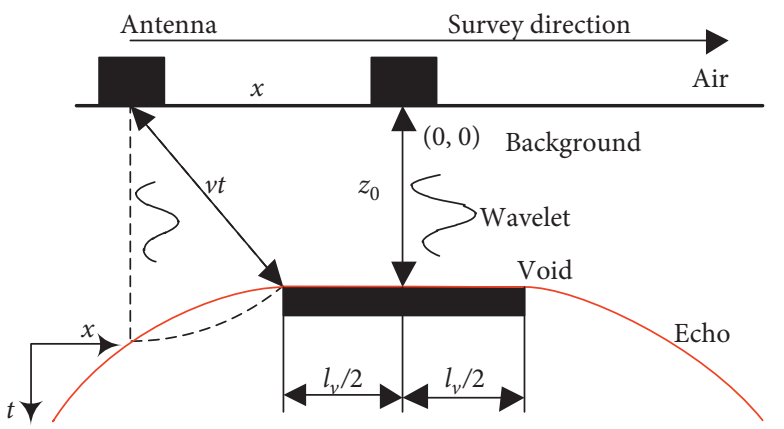

(b)

Figure 2: Rebar and void echo model: (a) rebar echo model; (b) void echo model.

longitudinal axis- $t$, which is also known as axis- $z$. The relationship between them is $t=2 z / v$. The longitudinal coordinate of the target is $z_{0}$. The symbol $v$ denotes the speed of the electromagnetic wave in the RC structure, $v=c / \sqrt{\varepsilon_{r}}$. When the antennas of the GPR locate $(x, 0)$, the two-way travel time of the rebar target is $t$.

The directional characteristics of the rebar echo in the space-time domain can be obtained from the differential of the echo signal relative to $x$ :

$$
S_{r}^{\prime}(x)=\frac{2 x}{\left(v \sqrt{x^{2}+z_{0}^{2}}\right)} .
$$

This indicates that the directional distribution of the rebar is along the hyperbolic, and the specific direction is determined by the propagation velocity, $v$. When $z_{0}=0$, the directional distribution can be expressed as $S_{r}^{\prime}(x)= \pm 2 / v$.

(2) Void Echo Model. Banded voids are the most typical defects found in RC structures. Figure 2(b) shows the echo of the rectangular void defect. The length of the void is $l_{v}$, and the intersection point between the void's central position and the direction of the survey line is the coordinate origin $O$ $(0,0)$. The rest of the settings are the same as in the rebar echo.

It can be seen from the graph that the void's echo is a piecewise function:

$$
S_{v}(x)= \begin{cases}\frac{2\left[\left(x+\left(l_{v} / 2\right)\right)^{2}+z_{0}^{2}\right]^{1 / 2}}{v}, & x \leq-\frac{l}{2}, \\ \frac{2 z_{0}}{v}, & -\frac{l}{2}<x<\frac{l}{2}, \\ \frac{2\left[\left(x-\left(l_{v} / 2\right)\right)^{2}+z_{0}^{2}\right]^{1 / 2}}{v}, & x \geq \frac{l}{2} .\end{cases}
$$

Similar to the rebar echo, the directional characteristics of the void echo in the space-time domain can be obtained from the differential of the echo signal relative to $x$ :

$$
S_{v}^{\prime}(x)= \begin{cases}\frac{2 x}{v \sqrt{x^{2}+z_{0}^{2}},} & x \longrightarrow-\infty, \\ 0, & -\frac{l}{2}<x<\frac{l}{2}, \\ -\frac{2 x}{v \sqrt{x^{2}+z_{0}^{2}},} & x \longrightarrow \infty .\end{cases}
$$

This indicates that the directional distribution characteristics of the banded void echoes are distributed along the hyperbolic and horizontal directions. When the GPR is located in the outer edge of a banded void, the directional 
distribution is characterized by the hyperbolic direction. When GPR is above the banded void, the directional distribution is characterized by the horizontal direction. When $z_{0}=0$, the directional distribution can be expressed as $s_{r}^{\prime}(x)= \pm 2 / v$, and the horizontal distribution as $s_{r}^{\prime}(x)=0$.

2.1.3. Spectral Analysis of Target Echo. Figure 2 and equations (4) and (6) describe the space-time distribution characteristics. To deduce the interference of the rebar echo, spectral analysis is conducted.

Generally, a GPR wavelet can be expressed as the Ricker wavelet:

$$
r(t)=\left(1-2 \pi^{2} f_{M}^{2} t^{2}\right) e^{-\pi^{2} f_{M}^{2} t^{2}}
$$

where $f_{M}$ represents the central frequency of the Ricker wavelet.

$$
R(f)=\frac{2}{\sqrt{\pi}} \frac{f^{2}}{f_{M}^{3}} e^{-\left(f^{2} / f_{M}^{2}\right)} .
$$

Figure 3 shows the time-frequency characteristics of the Ricker wavelet when $f_{M}=1.0 \mathrm{GHz}$.

Generally, the data sampling frequency of GPR is very high. For example, when the time window, $t_{w}$, is $8 \mathrm{~ns}$ and the number of samples is 512 , then the sampling frequency, $f_{s}$, is $64 \mathrm{GHz}$. Therefore, the spectrum of the Ricker wavelet lies in a low-frequency domain (the upper cut-off frequency of the Ricker wavelet is $2.5 \mathrm{GHz}$ when $f_{M}=1 \mathrm{GHz}$ ).

According to Figure 2, the echo profile (B-Scan) can be expressed as a convolution between $f(x, t)$ and the radar wavelet, $r(t)$ :

$$
g(x, t)=r(t) * f(x, t) .
$$

2.1.4. Spectral Characteristics of a Rebar Echo. Assume that the background medium is a lossless medium; i.e., the attenuation coefficient $\alpha=0$, and the phase shift coefficient $\beta=0$. The points on the rebar echo can be expressed using the Dirac function $f(x, t)$ :

$$
f(x, t)=r_{0} \delta\left(t-\frac{2 \sqrt{x^{2}+z_{0}^{2}}}{v}\right),
$$

where $r_{0}$ represents the reflection coefficient.

For simplification, assume that $z_{0}=0$; then the rebar echo is simplified into two fold lines. Taking the line left of the origin as an example, the slope of the line is $k=2 / v$, and equation (10) can be written as

$$
f(x, t)=r_{0} \delta\left(t-\frac{2 x}{v}\right) .
$$

Taking equation (11) into (9),

$$
g(x, t)=r(t) * r_{0} \delta\left(t-\frac{2 x}{v}\right) .
$$

A 2D Fourier transform is applied to both sides of equation (12) to obtain the following:

$$
G\left(\omega_{1}, \omega_{2}\right)=\iint g(x, t) e^{-j \omega_{1} x} e^{-j \omega_{2} t} \mathrm{~d} x \mathrm{~d} t .
$$

Simplifying equation (13), $G\left(\omega_{1}, \omega_{2}\right)$ can be written as

$$
G\left(\omega_{1}, \omega_{2}\right)=R\left(\omega_{2}\right) \delta\left(\omega_{1}+\frac{2 \omega_{2}}{v}\right) .
$$

Obviously, the spectrum of rebar void lies in the direction of $\omega_{2}=-v \omega_{1} / 2$, where this line is perpendicular to the line $t=2 x / v$.

2.1.5. Spectral Characteristics of a Void Echo. Similar to the rebar echo, the points on a void echo can be expressed using the Dirac function $f(x, t)$ and expressed as the following:

$$
S_{v}(x, t)= \begin{cases}r_{0} \delta\left(t+\frac{2\left[\left(x+\left(l_{v} / 2\right)\right)^{2}+z_{0}^{2}\right]^{1 / 2}}{v}\right), & x \leq-\frac{l}{2}, \\ r_{0} \delta(t), & -\frac{l}{2}<x<\frac{l}{2}, \\ r_{0} \delta\left(t-\frac{2\left[\left(x+\left(l_{v} / 2\right)\right)^{2}+z_{0}^{2}\right]^{1 / 2}}{v}\right), & x \geq \frac{l}{2} .\end{cases}
$$

Assume that $z_{0}=0$; then the void echo is simplified to three fold lines. Taking the left edge echo $(x \leq-l / 2)$ and horizontal echo $(-l / 2 \leq x \leq-l / 2)$ as examples, the spectral characteristics of the void echo can be analyzed.

(1) Spectrum of the Edge Echo. At the left edge, the slope is $k=2 / v$, and the echo can be expressed as

$$
f(x, t)=r_{0} \delta\left[t-\frac{2\left(x+\left(l_{v} / 2\right)\right)}{v}\right] .
$$

By combining equations (16) and (12), the edge echo can be written as

$$
g(x, t)=r(t) * r_{0} \delta\left[t-\frac{2\left(x+\left(l_{v} / 2\right)\right)}{v}\right] .
$$

A 2D Fourier transform can be applied to both sides of equation (17) to obtain the simplified equation:

$$
G\left(\omega_{1}, \omega_{2}\right)=R\left(\omega_{2}\right) \delta\left(\omega_{1}+\frac{2 \omega_{2}}{v}\right) e^{-j \omega_{1} l_{v} / v}
$$

It can be seen that the spectrum of the edge echo is equal to that of the rebar echo in amplitude, and to the $\omega_{1} l_{v} / v$ difference in phase.

(2) Spectrum of a Horizontal Echo. According to equation (15), a horizontal echo can be written as

$$
g(x, t)=r(t) * r_{0} \delta(t) .
$$

At this time, the horizontal echoes (where $k=0$ ) are exactly the same in the $x$ direction, so the spectrums of $r(t)$ 


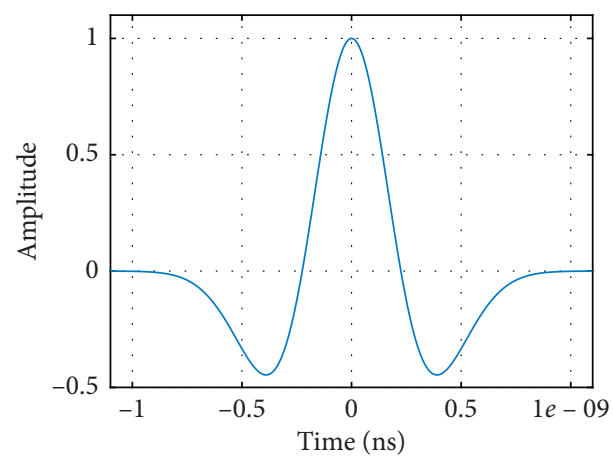

(a)

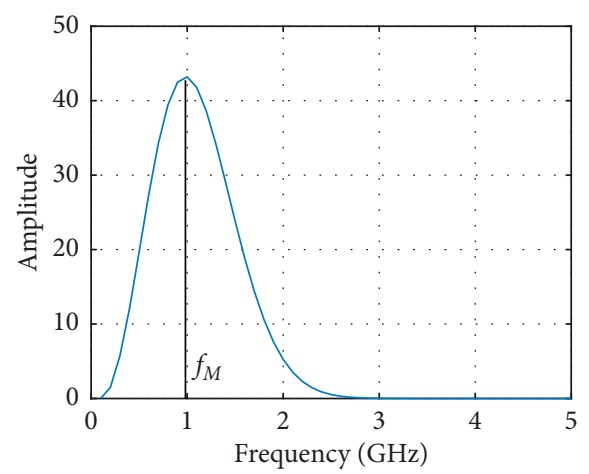

(b)

Figure 3: Time and frequency curve of the Ricker wavelet: (a) time domain; (b) frequency domain.

in the center frequency domain are identical, as shown below:

$$
G\left(\omega_{1}, \omega_{2}\right)=R\left(\omega_{2}\right)
$$

Figure 4 shows the spectral distribution of the target echo in different directions, where $k=0,1$, and 10 . When $k=0$, the direction of the spectral distribution is perpendicular to $\omega_{1}$, and the echo energy is located in the lowfrequency region. When $k$ increases, the direction of the spectral distribution is close to $\omega_{1}$, and the echo energy is inclined to the high-frequency region.

Based on the analysis above, the spectrum of a void echo in the horizontal portion is mainly distributed in the lowfrequency region. The spectrum of a rebar echo is mainly distributed in the high-frequency region. Therefore, an appropriate $2 \mathrm{D}$ low-pass filter would be useful to reduce the rebar echo.

2.1.6. Curvelet-Based Low-Pass Filter. GMA is a signal processing method proposed in the last ten years that overcomes the limitation of wavelet analysis. Wavelet analysis can only reflect the horizontal, vertical, and diagonal direction in two-dimensional data analysis, such as ridgelet and curvelet transforms, among others.

The curvelet transform was proposed by Candès in 2000, and two fast discrete curvelet transform methods were advocated in 2005. In the discrete curvelet transform, the radial window, $W(r)$, and the angular window, $V(t)$, are used to realize the multiscale analysis in the frequency domain. The high-frequency domain is called fine scale, and the low-frequency domain is called coarse scale. This is because the horizontal part of the void echo is located in the low-frequency domain in frequency. In this study, the coarse scale of the curvelet transform is regarded as a low-pass filter.

Assume that $c(j, l, k)$ is the curvelet coefficient,

$$
c(j, l, k):=\left\langle f, \varphi_{j, l, k}\right\rangle \text {, }
$$

where $j, l, k$ denote the scale, direction, and translate, respectively; $\varphi_{j, l, k}\left(x_{1}, x_{2}\right)$ denotes the mother curvelet function; and when $j=-1$, the scale is coarse scale.

The 2D low-pass filter is as follows:

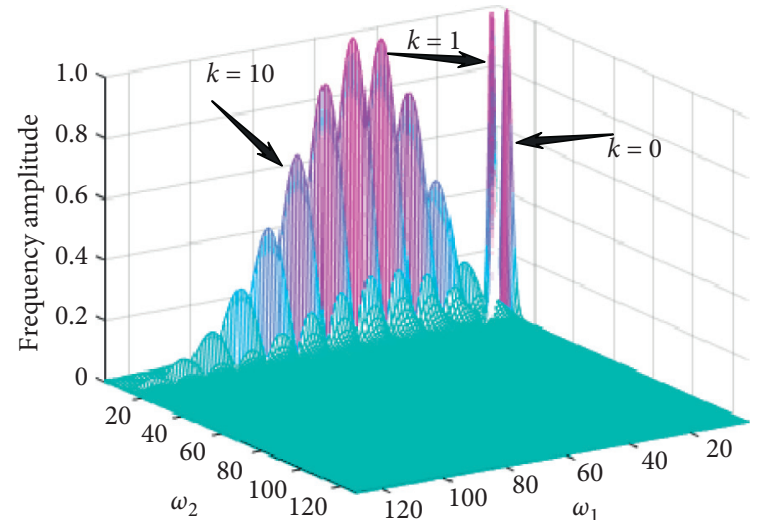

FIGURE 4: Spectral distribution in different directions.

$$
c^{\prime}(j, l, k)= \begin{cases}c(j, l, k), & j=-1, \\ 0, & \text { others. }\end{cases}
$$

Then, the reconstructed GPR profile $\tilde{f}\left(x_{1}, x_{2}\right)$ can be written as

$$
\tilde{f}\left(x_{1}, x_{2}\right)=\left\langle c^{\prime}(j, l, k), \varphi_{j, l, k}\left(x_{1}, x_{2}\right)\right\rangle .
$$

\subsection{RCS-Based Void-Size Determination Method. When the} low-pass filter is employed to reduce the interference of the rebar echo, the boundary of the void echo is eliminated too. Therefore, the void size cannot be identified directly using the method of energy interception.

When the void size is much smaller than its covered depth, it is believed that the distance from each point above the void defect to the receiving antenna of the GPR is identical, and the amplitude of the void echo is related to the radar cross section (RCS), as shown in Figure 5.

When the conductivity of the background medium is $\sigma=0$, the two typical relative positions between the antennas and the void defect are considered to be as follows:

(1) when the antenna is located at the edge of the void, $x_{0}$, and the RCS is proportional to $\theta_{0}$, $\theta_{0}=\tan ^{-1}\left(l_{v} / z\right)$ 


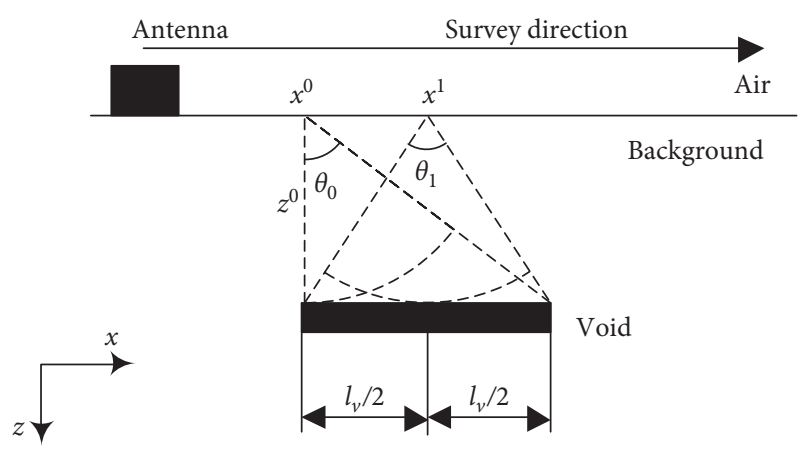

Figure 5: Target echo radar cross section.

(2) when the antenna is located at the top of the center of the void, $x_{1}$, the RCS is proportional to $\theta_{1}$, $\theta_{1}=2 \tan ^{-1}\left(l_{v} / 2 z\right)$

Obviously, from the Taylor expansion, $\theta_{1}$ is the maximum value. Therefore, a RCS-based regression function is constructed:

$$
y=a \tan ^{-1}\left(b l_{v}\right)+c,
$$

where $y$ denotes the amplitude of the central void echo and $a$, $b, c$ are the parameters.

However, due to the different power of the transmitting antenna, the attenuation coefficient of the background medium, and the covered depth of the void defect, the echo energy will be different. In this condition, the regression parameters $a, b$, and $c$ obtained by equation (24) are different. In order to eliminate this disadvantage, the echo amplitude of a certain void defect under the current working condition is often used as the reference value to normalize the whole data.

$$
y^{\prime}=\frac{y}{y_{0}}
$$

where $y_{0}$ denotes the amplitude of reference point, $y^{\prime}$ denotes the normalized amplitude, and regression parameters $a, b$, and $c$ combining equations (24) and (25) are the standard regression feature values.

\section{Simulation and Experiment}

To verify the time and frequency characteristics of the rebar echo and the void echo and to test the void-size determination method, forward simulation and experiment were carried out in different structural forms. The forward simulation was based on the Finite-Difference Time Domain (FDTD) [21, 22].

3.1. Void Defect Forward Simulation in Plain Concrete. A void defect model in plain concrete is shown in Figure 6. The size of the model is $1 \mathrm{~m} \times 0.55 \mathrm{~m}$. The location of the defect is $0.3 \mathrm{~m}$ on the $z$ axis, and the thickness is $h=3 \mathrm{~cm}$. The simulation parameters are shown in Table 1.

To compare the differences in void echoes with different lengths, the length, $l_{v}$, was set as variable, where $l_{v}=\{2,3,4$, $5,6,7,8,9,10,11 \mathrm{~cm}\}$. Figure 7 shows the forward

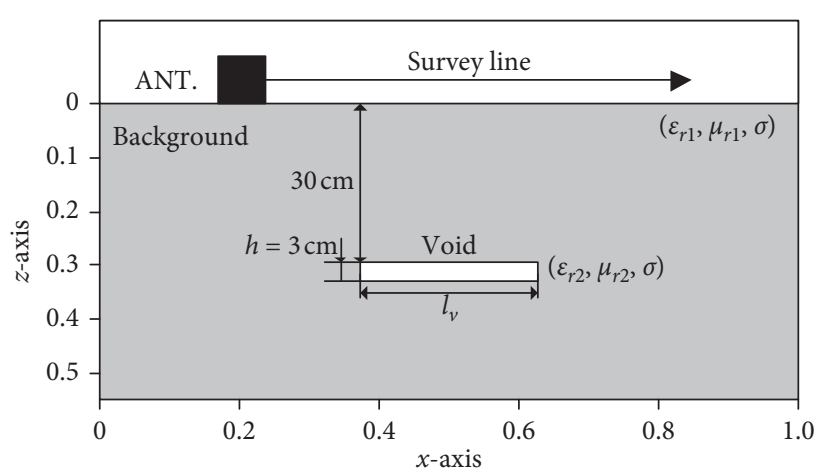

FIgure 6: Void defect model without rebar.

Table 1: Parameters of the forward simulation.

\begin{tabular}{lccc}
\hline Parameter & Value & Parameter & Value \\
\hline Grid & $(2 \mathrm{~mm}, 2 \mathrm{~mm})$ & Trace interval & $2 \mathrm{~mm}$ \\
Time window & $10 \mathrm{~ns}$ & Trace number & 350 \\
\hline
\end{tabular}

simulation and the processing results when $l_{v}=\{3,5,8$, $10 \mathrm{~cm}$. The removal of direct waves was not within the scope of this study, but usually a direct wave is removed directly using the time domain method. Therefore, 400-1600 sampling points were selected, as shown in Figure 7.

According to the forward and preprocessing results, with an increase in void size, the maximum value of the echo amplitude increased, which is consistent with the increase in the effective area of the echo reflection, as analyzed in Section 3.2. Also, from the result shown in Figures 7(e) and $7(\mathrm{f})$, the curvelet transform worked as a low-pass filter, effectively reducing the echo in the direction of the asymptote, but the edge features of the asymptote are not clear, which makes void-size recognition difficult. Figure 8 shows the distribution characteristics of the void echo at different $x$ points when the sample number equaled 878 (positive maximum point). Obviously, the edges of the void echo are blurred. The threshold normalization method showed no discernibility for the determination of void size, as shown in Figure 9.

According to Section 3.2, given that $l_{v}=\{2,4,6,8,10$, $11 \mathrm{~cm}\}$ are $x$, the corresponding maximum amplitudes are $y$. Regarding $(x, y)$ as training sets, and the echo amplitude $l_{v}=2 \mathrm{~cm}$ as the reference, the regression function is fitted. Given that $l_{v}=\{3,5,7,9 \mathrm{~cm}\}$ are $x_{1}$, the corresponding maximum amplitudes are $y_{1}$. Regarding $\left(x_{1}, y_{1}\right)$ as validation set, the fitting curve is shown in Figure 10. Table 2 shows the error under the condition of the regression function. It can be seen that when the void size is $10 \mathrm{~cm}$, the maximum determination error is $7.91 \%$.

3.2. Void Defect Forward Simulation in RC. The void defect model in a RC structure is shown in Figure 11 . The model size is $1.0 \mathrm{~m} \times 0.55 \mathrm{~m}$. The void defect lies $0.25 \mathrm{~m}$ in the structure and has a thickness $h=3 \mathrm{~cm}$. The void defect is covered by concrete and rebar, and the interval between the 


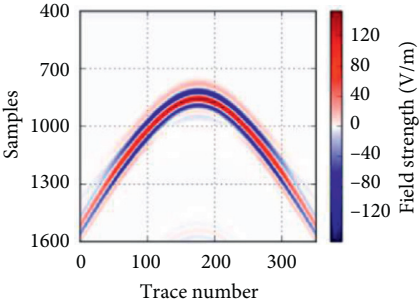

(a)

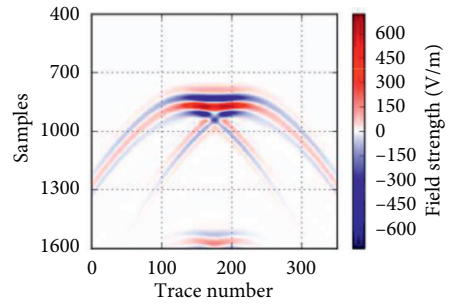

(d)

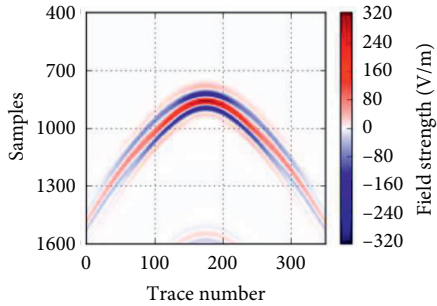

(b)

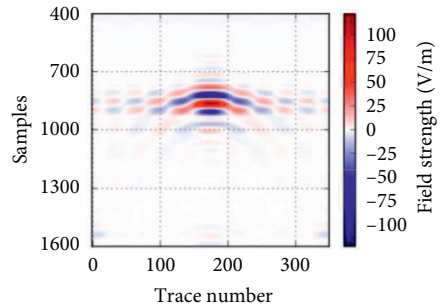

(e)

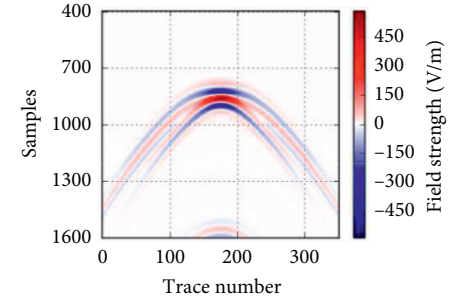

(c)

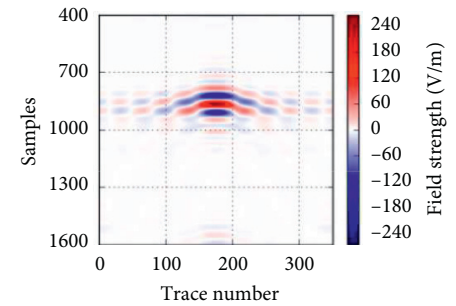

(f)

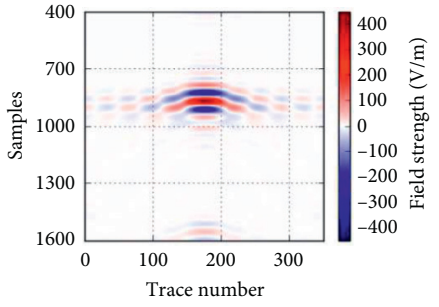

(g)

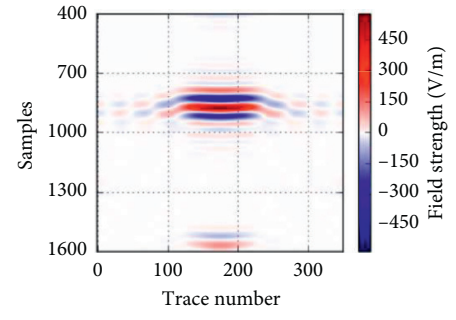

(h)

FiguRE 7: Forward and preprocessing results: (a) forward result, $l_{v}=3 \mathrm{~cm}$; (b) forward result, $l_{v}=5 \mathrm{~cm}$; (c) forward result, $l_{v}=8 \mathrm{~cm}$; (d) forward result, $l_{v}=10 \mathrm{~cm}$; (e) preprocessing result of (a); (f) preprocessing result of (b); (g) preprocessing result of (c); (h) preprocessing result of (d).

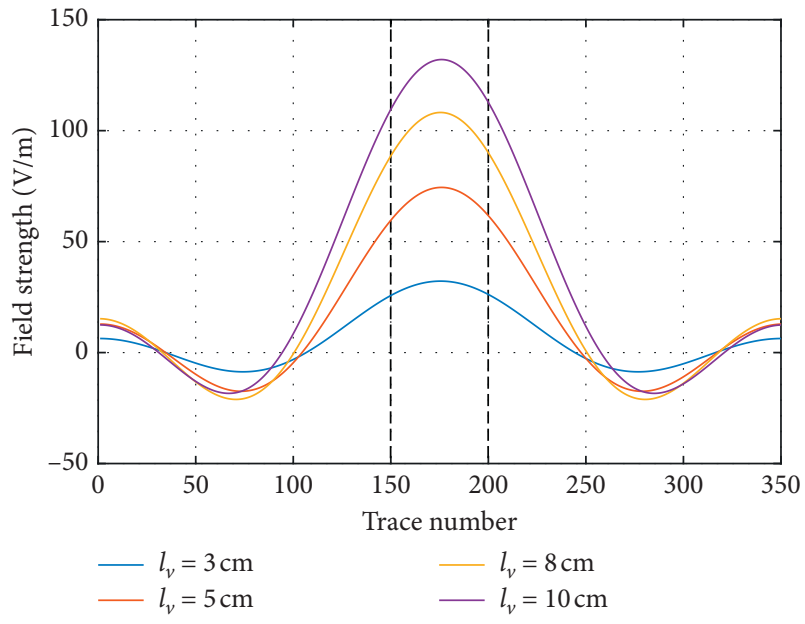

Figure 8: Void echo space distribution in sample number $=878$.

rebar is $20 \mathrm{~cm}$, with distance to the ground of $10 \mathrm{~cm}$. Similar to Section 3.1, the length of $l_{v}$ is set as variable, where $l_{v}=\{2$, $3,4,5,6,7,8,9,10,11 \mathrm{~cm}\}$. Figure 12 shows the forward and preprocessing results when $l_{v}=\{3,5,8,10 \mathrm{~cm}\}$.

In Figures 12(a) and 12(b), the void size is relatively small, and the rebar echo is the maximum value of $450 \mathrm{~V} / \mathrm{m}$. In Figures $12(\mathrm{c})$ and 12(d), with an increase in void size, the void

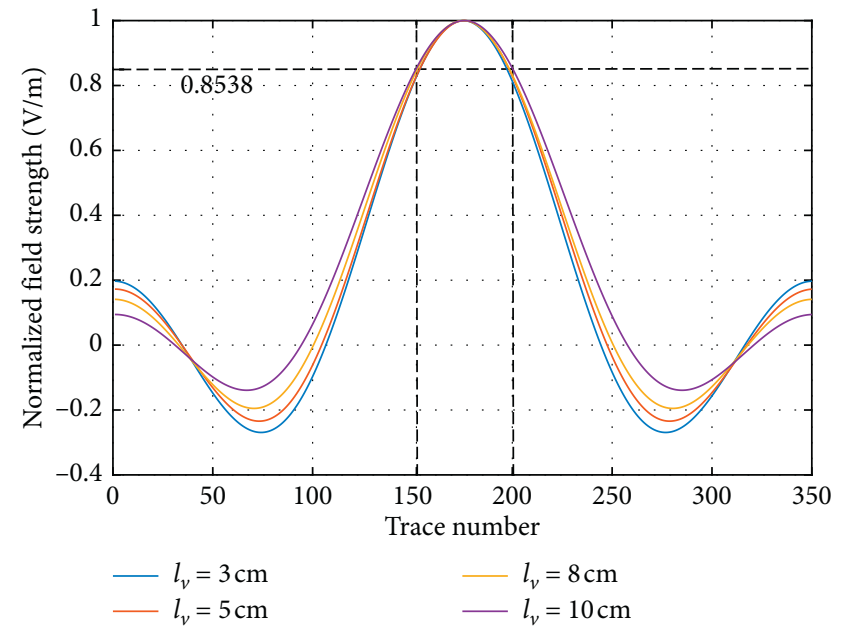

FIgURE 9: Threshold-based method to determine void size.

echo is the maximum value when $l_{v}=8 \mathrm{~cm}$, and the amplitude is $578.8 \mathrm{~V} / \mathrm{m}$. When $l_{v}=10 \mathrm{~cm}$, the amplitude is $621.2 \mathrm{~V} / \mathrm{m}$.

Figures 12(e) through 12(h) show the preprocessing results of Figures 12(a) through 12(d). Obviously, the void defect exists near sample point 1000. But due to the low-pass filter, the preprocessing result is similar, and it is hard to determine the void size directly. 


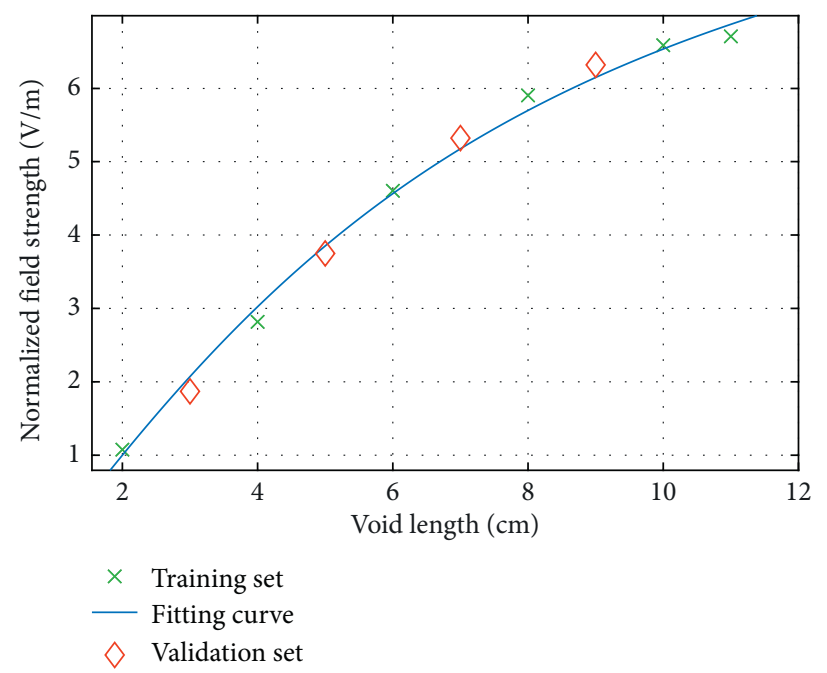

Figure 10: Void size-echo amplitude regression curve of plain concrete.

TABLE 2: Void-size determination errors in plain concrete.

\begin{tabular}{lcc}
\hline Void size $(\mathrm{cm})$ & Estimated value $(\mathrm{cm})$ & Error $(\%)$ \\
\hline 2 & 2.00 & 0.00 \\
3 & 2.80 & -6.55 \\
4 & 3.77 & -5.71 \\
5 & 4.87 & -2.65 \\
6 & 6.06 & 0.98 \\
7 & 7.26 & 3.75 \\
8 & 8.44 & 5.45 \\
9 & 9.42 & 4.70 \\
10 & 10.14 & 1.41 \\
11 & 10.49 & -4.62 \\
\hline
\end{tabular}

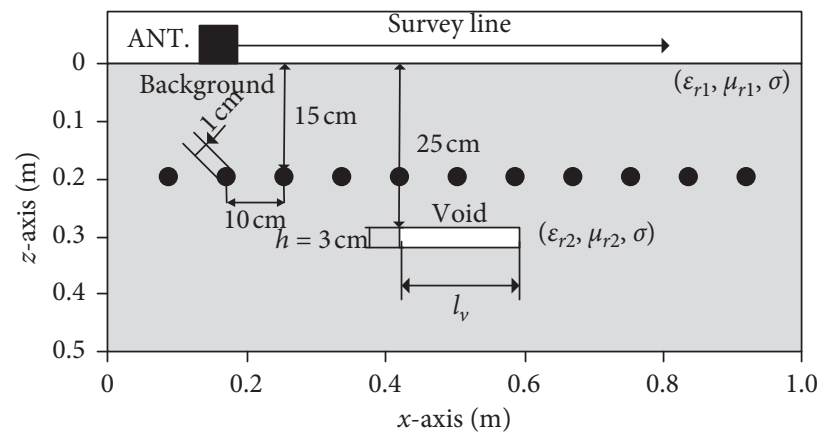

Figure 11: Void defect model with rebar.

Similar to Section 3.1, regarding the echo amplitude $l_{v}=2 \mathrm{~cm}$ as the reference, the forward and preprocessing results were divided into two sets: a training set and validation set. Then a regression process was executed, as shown in Figure 13. Table 3 shows the errors of the regression function. It can be seen that when the void size is $4 \mathrm{~cm}$, the maximum determination error is $-6.75 \%$. Comparing Figure 10 with Figure 13, the regression curves show significant similarity. Therefore, the parameters $a, b$, and $c$ can be regarded as standard regression feature values.
3.3. Void Defect in Experiment. To further verify the regression characteristics of cavity defect echo, experiment was carried out. In the experiment, sand is used instead of concrete, and polystyrene foam is used to simulate void defects. The length of void $l_{v}=\{2,3,4,6,8,10 \mathrm{~cm}\}$, as shown in Figure 14(a). The void lies in the lower layer of sand and is covered by rebar. Figures 14(d) and 14(e) show the test result and the filtered data. And regarding the echo amplitude $l_{v}=2 \mathrm{~cm}$ as the reference, the normalized field strength was obtained as shown in Figure 14(f). Meanwhile, the regression 


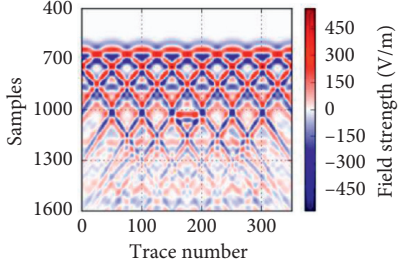

(a)

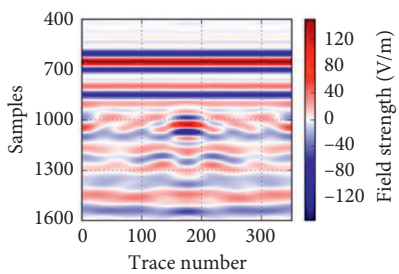

(e)

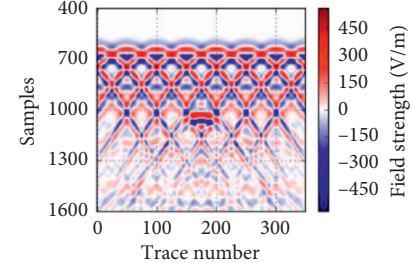

(b)

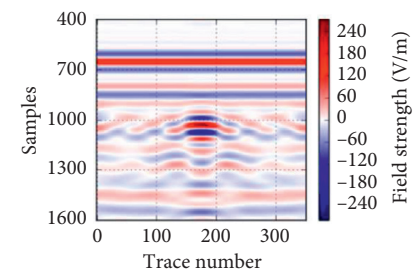

(f)

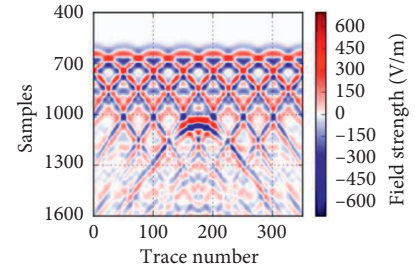

(c)

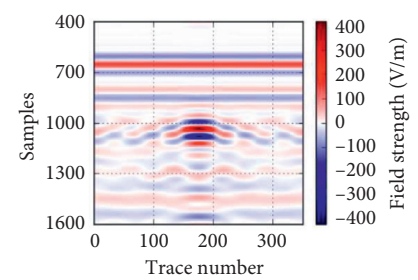

(g)

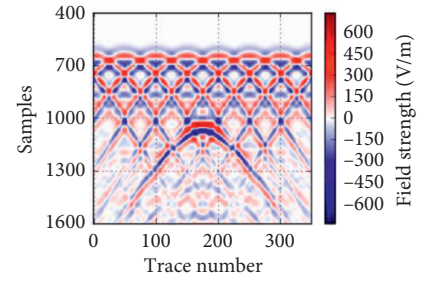

(d)

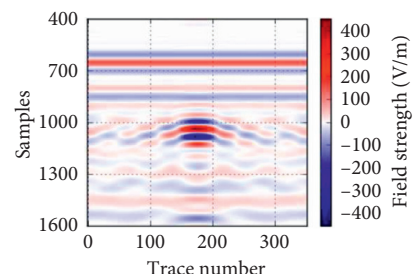

(h)

FIGURE 12: Forward and preprocessing results for rebar: (a) forward result, $l_{v}=3 \mathrm{~cm}$; (b) forward result, $l_{v}=5 \mathrm{~cm}$; (c) forward result, $l_{v}=8 \mathrm{~cm}$; (d) forward result, $l_{v}=10 \mathrm{~cm}$; (e) preprocessing result of (a); (f) preprocessing result of (b); (g) preprocessing result of (c); (h) preprocessing result of $(\mathrm{d})$.

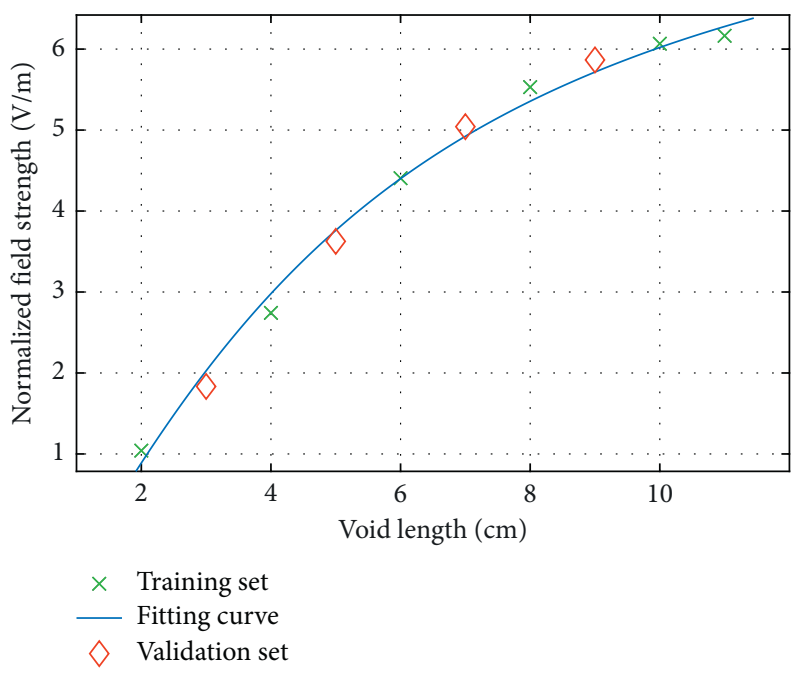

Figure 13: Void size-echo amplitude regression curve of RC structure.

TABLE 3: Void-size determination errors of the rebar.

\begin{tabular}{lcc}
\hline Void size $(\mathrm{cm})$ & Estimated value $(\mathrm{cm})$ & Error $(\%)$ \\
\hline 2 & 2.00 & 0.00 \\
3 & 2.82 & -6.00 \\
4 & 3.73 & -6.75 \\
5 & 4.81 & -3.80 \\
6 & 6.01 & 0.17 \\
7 & 7.26 & 3.71 \\
8 & 8.46 & 5.75 \\
9 & 9.47 & 5.22 \\
10 & 10.16 & 1.60 \\
11 & 10.53 & -4.27 \\
\hline
\end{tabular}

curve is drawn in Figure 14(f). Table 4 shows the errors under the condition of the regression feature values $a, b$, and $c$ obtained in Section 2.2. Obviously, when the void size is small, the estimation error is small. With an increase in void size, the estimation error increased. When the void size is $10 \mathrm{~cm}$, the maximum determination error reaches $-18.70 \%$. 

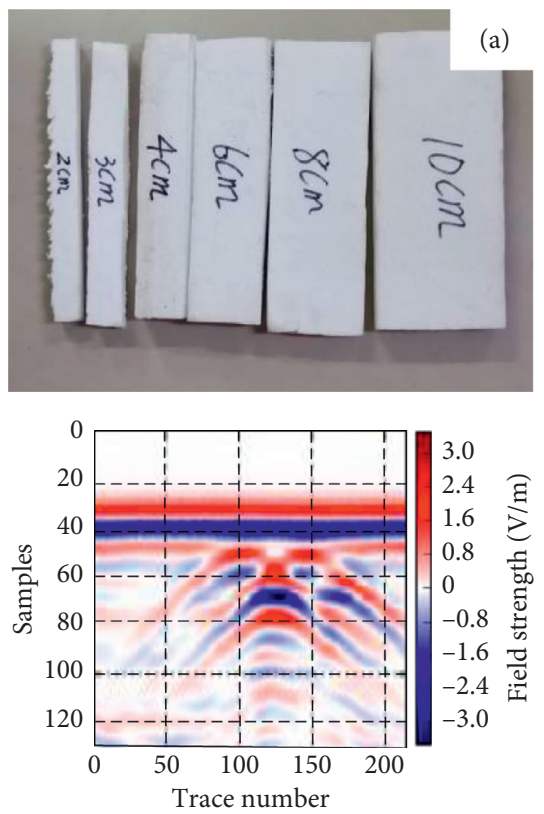

(d)
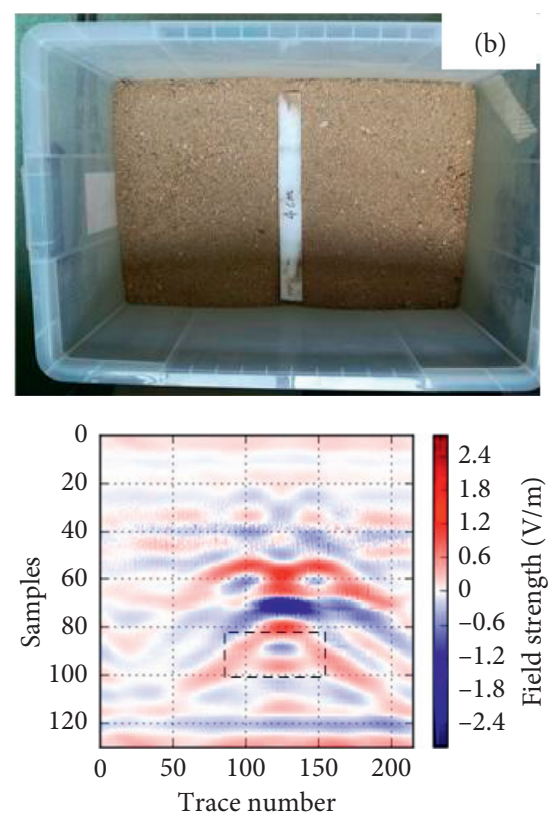

(e)
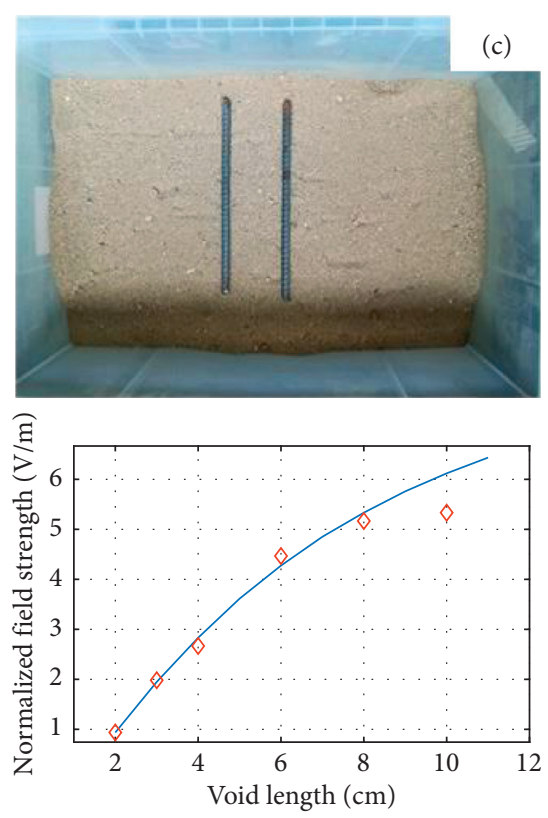

- Regression curve

$\diamond$ Experimental data

(f)

FIGURE 14: Experiment and processing result: (a) experimental void defects; (b) void defect in lower layer; (c) rebar in upper layer; (d) test result when void length $l_{v}=6 \mathrm{~cm}$; (e) filtered result of (d); (f) comparison between regression curve and experimental data.

TABle 4: Void-size determination errors in experiment.

\begin{tabular}{lcc}
\hline Void size $(\mathrm{cm})$ & Estimated value $(\mathrm{cm})$ & Error $(\%)$ \\
\hline 2 & 2.00 & 0 \\
3 & 3.21 & 7.00 \\
4 & 3.62 & -9.50 \\
6 & 6.02 & -0.33 \\
8 & 7.25 & -9.38 \\
10 & 8.13 & -18.70 \\
\hline
\end{tabular}

\section{Conclusion}

Based on spectral analysis and the relationship investigation between echo amplitude and dimension characterization of small-size void, a two-stage regression method was proposed to develop the tactic of GPR-based small-scale void-size determination in RC structure, and the main conclusions are as follows:

(1) The strong reflection interference of the rebar echo is the main obstacle in the identification of a void defect in RC structures. Spectrum analysis showed that the echo frequency of the rebar is mainly distributed in the high-frequency portion of the spectrum; otherwise, the frequency of the central part of the void echo is mainly distributed in the low-frequency portion.

(2) The difference between the rebar echo and the void echo indicates that it is possible to be denoised by filtering out the high-frequency components in the GPR signal. The low-pass filter based on the curvelet transform is an effective tool to reduce the interference of the rebar echo.

(3) The regression method based on RCS performs well in dimension characterization, whose estimation error is less than $10 \%$ for voids less than $10 \mathrm{~cm}$, no matter for plain concrete structures or reinforced concrete structures.

This proposed method is reliable for the identification of small-size void and is conducive to broadening the prospects about the application and promotion of the GPR-based nondestructive techniques for multilayer reinforced concrete structures.

\section{Data Availability}

The data used to support the findings of this study are available from the corresponding author upon request.

\section{Conflicts of Interest}

The authors declare that they have no conflicts of interest regarding the publication of this paper.

\section{Acknowledgments}

This research was financially funded by the National Natural Science Foundation of China (no. 51978423), Natural Science Foundation in Hebei Province (nos. E2019210214, 19210804D, and E2016210104), and Department of Education of Hebei Province of China (no. ZD2020311). 


\section{References}

[1] X. Tian, W. Zhao, Y. Du, and B. Wang, "Detection of mortar defects in ballastless tracks of high-speed railway using transient elastic wave method," Journal of Civil Structural Health Monitoring, vol. 8, no. 1, pp. 151-160, 2018.

[2] O. Abraham, C. Leonard, and P. Cote, "Time frequency analysis of impact-echo signals: numerical modeling and experimental validation," ACI Structural Journal, vol. 97, no. 6 , pp. $645-656,2000$.

[3] A. Robert, "Dielectric permittivity of concrete between $50 \mathrm{MHz}$ and $1 \mathrm{GHz}$ and gpr measurements for building materials evaluation," Journal of Applied Geophysics, vol. 40, no. 1-3, pp. 89-94, 1998.

[4] J. Hugenschmidt and R. Mastrangelo, "GPR inspection of concrete bridges," Cement and Concrete Composites, vol. 28, no. 4, pp. 384-392, 2006.

[5] R. Ahmadi and N. Fathianpour, "Estimating geometrical parameters of cylindrical targets detected by ground-penetrating radar using template matching algorithm," Arabian Journal of Geosciences, vol. 10, no. 6, pp. 140-152, 2017.

[6] L. Zanzi and D. Arosio, "Sensitivity and accuracy in rebar diameter measurements from dual-polarized gpr data," Construction and Building Materials, vol. 48, no. 19, pp. 1293-1301, 2013.

[7] K. Hoegh, L. Khazanovich, S. Dai, and T. Yu, "Evaluating asphalt concrete air void variation via gpr antenna array data," Case Studies in Nondestructive Testing and Evaluation, vol. 3, pp. 27-33, 2015.

[8] W. He, R. Wu, and J. Liu, "Void-layer detection and depth determination in runways based on gpr," Signal Processing, vol. 1, no. 10, pp. 182-185, 2011.

[9] J. Brian, R. Alastair, M. Jennifer, and W. Patricia, "Detecting voids within a historical building façade: a comparative study of three high frequency GPR antenna," Journal of Cultural Heritage, vol. 32, 2018.

[10] D. C. Nobes, "Ground penetrating radar response from voids: a demonstration using a simple model," NDT \& E International, vol. 91, pp. 47-53, 2017.

[11] Z. Mechbal and A. Khamlichi, "Determination of concrete rebars characteristics by enhanced post-processing of GPR scan raw data," NDT \& E International, vol. 89, pp. 30-39, 2017.

[12] C. G. Windsor, L. Capineri, and P. Falorni, "The estimation of buried pipe diameters by generalized hough transform of radar data," Piers Online, vol. 1, no. 3, pp. 345-349, 2005.

[13] W. Al-Nuaimy, Y. Huang, M. Nakhkash, M. T. C. Fang, V. T. Nguyen, and A. Eriksen, "Automatic detection of buried utilities and solid objects with GPR using neural networks and pattern recognition," Journal of Applied Geophysics, vol. 43, no. 2-4, pp. 157-165, 2000.

[14] E. Pasolli, F. Melgani, and M. Donelli, "Automatic analysis of gpr images: a pattern-recognition approach," IEEE Transactions on Geoscience and Remote Sensing, vol. 47, no. 7, pp. 2206-2217, 2009.

[15] E. Pasolli, F. Melgani, and M. Donelli, "Gaussian process approach to buried object size estimation in gpr images," IEEE Geoscience and Remote Sensing Letters, vol. 7, no. 1, pp. 141-145, 2010.

[16] E. Candès, L. Demanet, D. Donoho, and L. Ying, "Fast discrete curvelet transforms," Multiscale Modeling \& Simulation, vol. 5, no. 3, pp. 861-899, 2006.
[17] J. L. Starck, E. J. Candès, and D. L. Donoho, "The curvelet transform for image denoising," IEEE Transactions on Image Processing, vol. 11, no. 6, pp. 670-684, 2002.

[18] J. Ma and G. Plonka, “The curvelet transform," IEEE Signal Processing Magazine, vol. 27, no. 2, pp. 118-133, 2010.

[19] A. Tzanis, "A versatile tuneable curvelet-like directional filter with application to fracture detection in two-dimensional gpr data," Signal Processing, vol. 132, pp. 243-260, 2017.

[20] A. Tzanis, "Detection and extraction of orientation-and-scaledependent information from two-dimensional gpr data with tuneable directional wavelet filters," Journal of Applied Geophysics, vol. 89, pp. 48-67, 2013.

[21] J. P. Renger, "Perfectly matched layer for the FDTD solution of wave-structure interaction problems," IEEE Trans on Antennas \& Propagation, vol. 44, no. 1, pp. 110-117, 1996.

[22] C. Warren, A. Giannopoulos, and I. Giannakis, "gprMax: open source software to simulate electromagnetic wave propagation for ground penetrating radar," Computer Physics Communications, vol. 209, pp. 163-170, 2016. 